\title{
Experiencia sobre sledd en cuidados intensivos
}

\author{
Nuno João dos Santos Caramelo \\ Jesus Domínguez Fernández
}

Diplomados en Enfermería

Unidad de Cuidados Intensivos

Hospital de Curry Cabral. Lisboa

\begin{abstract}
RESUMEN
El uso de técnicas dialíticas mixtas como la SLEDD (Slow low efficient daily dialysis) ofrece numerosos beneficios que pueden ser muy útiles en las unidades de cuidados intensivos, dado que combinan las ventajas de la hemodiálisis convencional (intermitente) y las técnicas dialíticas continuas.
\end{abstract}

En la técnica SLEDD sometemos a un paciente a una "hemodiálisis" en la que se alteran ciertas características, como son entre otras, la duración del procedimiento, el flujo del dializante y la velocidad de la bomba de sangre. Todo esto se hace con la pretensión de adaptar la técnica a la delicada situación hemodinámica de los pacientes y con el objetivo de alcanzar en un periodo de tiempo menor al de las técnicas continuas, una igual o mayor depuración de urea y creatinina, y un mayor volumen de líquido ultrafiltrado.

Se describe en este trabajo una experiencia de implementación de SLEDD, comparándola con una técnica continua, en una unidad de cuidados intensivos. Se demostró que aporta beneficios para el paciente en términos fisiológicos, ventajas para el personal de enfermería y también ventajas de tipo económico.

Las tasas medias de aclaramiento de urea y creatinina de la SLEDD son superiores a las de las técnicas continuas, y las consecuencias de esta técnica mixta en términos hemodinámicos para el paciente, son similares y a veces menos descompensadoras que las técnicas continuas, incluso en situaciones en las que existe soporte de aminas.

\begin{tabular}{c} 
Correspondencia: \\
Jesus Domínguez Fernández \\
Rua Diogo Bernardes №20, 2ํo Frente. \\
2700 Amadora \\
PORTUGAL \\
\hline
\end{tabular}

\author{
PALABRAS CLAVE: SLEDD (Slow low efficient daily dialysis) \\ TÉCNICAS DIALÍTICAS CONTINUAS \\ COSTES
}

\section{THE EXPERIENCE OF SLEDD IN PATIENTS IN INTENSIVE CARE}

\begin{abstract}
The use of mixed dialytic techniques such as SLEDD (Slow low efficient daily dialysis) offers multiple benefits that may be very useful in intensive care units, as they combine the advantages of conventional haemodialysis (intermittent) and continuous dialytic techniques.
\end{abstract}

In the SLEDD technique, we will submit a patient to a "haemodialysis" where certain characteristics are altered, such as the duration of the procedure, the flow of the dialisant and the blood pumping speed. All of this is done to adapt the technique to the delicate haemodynamic situation of the patients and in order to achieve at shorter times than with continuous techniques, the same or greater purification of urea and creatinine and a larger volume of ultrafiltered liquid.

This work describes an experimental implementation of SLEDD, comparing it with a continuous technique in an intensive care unit. It was shown that it gives benefits for the patient in physiological terms, advantages for nursing personnel and also economic advantages.

The mean urea and creatinine clarification rates of SLEDD are better than those of continuous techniques, and the consequences of this mixed technique in haemodynamic terms for patients are similar and sometimes less decompensating than the continuous techniques, even in situations where there is amine support. 
KEYWORDS: SLEDD (Slow low efficient daily dialysis) CONTINUOUS DIALYTIC TECHNIQUES COSTS

\section{INTRODUCCIÓN}

Para realizar la técnica dialítica SLEDD (Slow low efficient daily dialysis) se utiliza un monitor convencional de hemodiálisis y se programa un tiempo de duración y otras características adaptadas al paciente crítico. En esta técnica pretendemos someter al paciente a una "hemodiálisis" de 6 a 12 horas de duración, con la intención de sustituir a una técnica de tipo continua (durante 24 horas) y manteniendo, dentro de lo posible, un mejor equilibrio y mejores resultados de aclaramiento/filtración de productos de desecho, en pacientes cuya inestabilidad hemodinámica, no permite practicar una hemodiálisis convencional. Pretendemos, por tanto, realizar una "técnica mixta" en la que se unan las ventajas de la hemodiálisis convencional y de las técnicas continuas.

Partimos del principio de que al paciente afecto de insuficiencia renal aguda, dado su grave estado general y su situación hemodinámica, no se le puede realizar una técnica de depuración intermitente (hemodiálisis convencional) para eliminar los productos de desecho que normalmente son filtrados por el riñón. Habitualmente en estos casos en las unidades de cuidados intensivos se utilizan técnicas continuas como la hemodiafiltración (HDVVC), con el fin de evitar grandes alteraciones del estado hemodinámico de los pacientes. Sabemos que las técnicas continuas tienen inconvenientes debido, entre otros motivos, a la exposición de forma continua de la sangre del paciente a un circuito extracorpóreo y a una solución dializante y de reposición que están a temperatura ambiente, lo que puede provocar alteraciones en su temperatura corporal $y$, por tanto, derivar en alteraciones que afecten a la perfusión de los tejidos y al gasto cardiaco, así como otras complicaciones relacionadas con la anticoagulación.

Con la SLEDD pretendemos ajustar los parámetros de un monitor convencional de hemodiálisis a la situación critica en la que se encuentra el paciente, es decir, vamos a preparar unas condiciones en las que al paciente se le va a practicar una filtración de los productos de desecho y pérdida de exceso de líquidos, por medio de los principios de la diálisis normal, sin afectar de manera importante su situación hemodinámica.

Según Kumar y otros autores, se produjeron similares situaciones de descompensación hemodinámica en pacientes que realizaron SLEDD que en técnicas de hemodiafiltración continua. Además, se encontraron incluso mejores resultados en términos de aclaramiento de creatinina y urea después de 24 horas, ya que, según este autor, para conseguir niveles ade- cuados de estos productos con una técnica continua es necesario someter al paciente de 48 a 72 horas de filtración, debido a la menor eficacia de estas técnicas continuas para ultrafiltrar los productos de desecho. La técnica de SLEDD resultó incluso más eficaz que la hemodiálisis convencional (intermitente) en pacientes ingresados en cuidados intensivos.

En las técnicas hemodialíticas se debe prestar especial atención a los anticoagulantes administrados ya que desde el punto de vista de la anticoagulación en pacientes inestables, las alteraciones derivadas del exceso de anticoagulante pueden tener consecuencias fatales. Según algunos estudios, el 10\% de las muertes en cuidados intensivos se relacionan con problemas de coagulación. En una técnica de tipo continua, la anticoagulación, en principio, también va a ser continua, aumentando el riesgo para el paciente. Según Kumar, un 31\% de los pacientes que fueron sometidos a SLEDD, necesitaron cantidades menores de anticoagulantes, o incluso no los precisaron, en contraste con pacientes que realizaron técnicas continuas. Por otra parte, el filtro usado en las técnicas continuas va a sufrir un deterioro progresivo por causa de los depósitos de fibrina que se van acumulando en él a lo largo del tiempo, disminuyendo así la eficacia de la técnica progresivamente. En la SLEDD, la técnica transcurre en un periodo de 6 a 12 horas, la exposición a anticoagulante, evidentemente, es menor y con ello también las complicaciones derivadas de la misma.

Para la técnica SLEDD, el uso de un monitor convencional de diálisis puede ofrecer un mejor control de flujo del líquido dializante y de la temperatura del mismo, lo que se traduce en mejor control de la temperatura y hemodinamia del paciente, mejor monitorización del procedimiento y mejor control de la conductividad del líquido, entre otras ventajas. Otras necesidades técnicas de SLEDD son: la preparación del material, el cebado de las líneas del circuito extracorpóreo, la calibración, la conexión y desconexión del paciente, las complicaciones referidas tanto al paciente como al procedimiento. En resumen, el manejo y manipulación del monitor será similar al realizado para una máquina de hemodiálisis convencional, aunque existen diferencias como el débito de la bomba de sangre, el flujo del líquido dializante y el volumen ultrafiltrado por hora al paciente, que serán menores que en una diálisis convencional, mientras que el tiempo del procedimiento es de 6 a 12 horas.

Al utilizar un monitor convencional se evitan muchos problemas relacionados con el uso de los monitores de hemodiafiltración continua como: el recambio de bolsas de dializante cada cierto tiempo, dificultades con las balanzas que pueden dar problemas de descalibración y por consiguiente tener que reiniciar la técnica. Cabe mencionar que la exposición constante de la sangre del paciente al medio "externo" durante la realización de las técnicas contínuas y los elevados costos del material en relación a los necesarios para una hemodiálisis con- 
vencional, hacen que las técnicas mixtas sean un buen criterio de elección en lo que respecta a terapias sustitutivas de función renal en cuidados intensivos.

\section{OBJETIVOS}

1. Evaluar eficacia de la técnica SLEDD en la unidad de cuidados intensivos, en términos de depuración de urea y creatinina.

2. Identificar las nuevas necesidades/requerimientos para la adecuación de los enfermeros a esta experiencia e identificar ventajas sobre las otras técnicas dialíticas realizadas en la unidad.

3. Confirmar que las técnicas mixtas reportan menores gastos que las técnicas de tipo continuas.

\section{PACIENTES Y MÉTODO}

En base a los conocimientos anteriormente expuestos, decidimos realizar una experiencia sobre SLEDD en la unidad de cuidados intensivos donde trabajamos y compararla con las técnicas continuas que realizamos en el mismo periodo de tiempo.

La unidad de cuidados intensivos donde se realizó esta experiencia, cuenta en cada equipo con un mínimo de 2 a 3 personas con conocimientos fluidos en hemodiálisis, así como en técnicas continuas de sustitución de la función renal; cuenta también con dos máquinas de hemodiálisis convencional y sus centrales de tratamiento de aguas portátiles, así como una maquina de hemodiafiltración continua de última generación.

El estudio de SLEDD se inició en el último trimestre del 2003, completándose a los 6 meses. Se incluyeron 12 pacientes (7 hombres y 5 mujeres), edad media 60,3 años, clasificados como pacientes críticos en APACHE II (media 29.8) y SAPS II (media 56). Se realizaron un total de 37 procedimientos; de estos 12 pacientes, 6 se encontraban bajo soporte de aminas (20 procedimientos del total), y todos estaban sometidos a ventilación asistida.

El número de horas de diálisis en cada sesión fue de media de 6,8 horas, con un intervalo de 6 a 10 horas, utilizando siempre el mismo tipo de filtro convencional. La velocidad media de la bomba fue de $150 \mathrm{cc} / \mathrm{min}$, con un intervalo de $120 \mathrm{a}$ $200 \mathrm{cc} / \mathrm{min}$, y flujo de dializante constante de $500 \mathrm{cc} / \mathrm{min}$. La cantidad de heparina de inicio fue de 1000 UI y la heparina horaria de $500 \mathrm{UI} /$ hora, prescribiéndose heparinas de bajo peso molecular en pocos casos. El volumen de líquido extraído a los pacientes fue de 0 a 3500 cc, con una media de 1930 cc.
La evaluación de la eficacia de la técnica se hizo en base a la tasa de filtración de urea y de creatinina (URR y CRR), comparándolas posteriormente con las tasas de filtración de una técnica continua (HDVVC).

En relación a las técnicas continuas que fueron realizadas en el mismo periodo de tiempo se estudiaron 6 pacientes ( 4 hombres y 2 mujeres), con una edad media de 56,7 años. Según la clasificación de pacientes críticos APACHE II (media de 30,2) y SAPS II (media de 57,3). En estos pacientes fueron realizados un total de 14 procedimientos; 4 de los pacientes tenían soporte de aminas, siendo realizados en estas circunstancias 8 procedimientos.

Cada procedimiento tuvo una duración media de 26,4 horas, con una velocidad de bomba media de $130 \mathrm{cc} / \mathrm{min}$ (100 y $150 \mathrm{cc} / \mathrm{min})$. Heparina horaria $500 \mathrm{UI} /$ hora. Volumen de líquido extraído en 24 horas entre 2400 y 3600 cc (media $2890 \mathrm{cc}$ ). Con flujo dializante de $1000 \mathrm{cc} /$ hora. Se evaluaron los niveles de urea y creatinina antes del inicio de la técnica y a las 24 horas, y las tasas de aclaramiento.

\section{RESULTADOS}

\section{Resultados de SLEDD}

Los valores de urea y creatinina obtenidos inmediatamente antes y una hora después de finalizar cada procedimiento, se describen en la tabla 1. En relación a los datos obtenidos la urea media antes del inicio de los procedimientos es de 163,7 $\mathrm{mg} / \mathrm{dl}$. La urea media, obtenida una hora después de acabar cada técnica es de $84,5 \mathrm{mg} / \mathrm{dl}$, siendo la media de la tasa de aclaramiento de urea de 48,3\%.

Con respecto a la creatinina la media pre-procedimiento es de $3,9 \mathrm{mg} / \mathrm{dl}$, siendo después del procedimiento de 2,2 $\mathrm{mg} / \mathrm{dl}$; la tasa media de aclaramiento de creatinina es del $43 \%$.

\section{Resultados de técnica continua}

Los valores obtenidos con la técnica continua se reflejan en la tabla 2 , obteniéndose un valor medio de urea pre-técnica de $226,5 \mathrm{mg} / \mathrm{dl}$, y de $144 \mathrm{mg} / \mathrm{dl}$ después de 24 horas de técnica, siendo la tasa de aclaramiento media del $37 \%$.

En relación a la creatinina, tenemos un valor pre-técnica medio de 5,2 mg/dl, de 3,55 mg/dl después de 24 horas y una tasa media de aclaramiento de creatinina del 31,7 \%. Las tasas medias del aclaración de urea y creatinina de ambas técnicas se reflejan en la tabla 3 .

\section{Comparación de costos de ambas técnicas}

En relación a los costos materiales, una hemodiafiltración veno-venosa continua resulta ser más cara que la técnica SLEDD. En una sesión de SLEDD gastamos aproximadamen- 
te en el filtro 11 euros, en las líneas del circuito 4,50 euros, en bicarbonatos 12 euros y en kits de desinfección 10 euros, lo que hace un total de 37,50 euros.

\begin{tabular}{|c|c|c|c|}
\hline urea pre & urea post & Creat pre & Creat post \\
\hline 166 & 61 & 5.8 & 2.6 \\
\hline 163 & 85 & 5.8 & 3.8 \\
\hline 116 & 42 & 4.8 & 2.3 \\
\hline 232 & 135 & 6.5 & 4.6 \\
\hline 264 & 107 & 7 & 3.8 \\
\hline 188 & 67 & 5.2 & 2.9 \\
\hline 67 & 58 & 2.9 & 2.7 \\
\hline 122 & 103 & 4 & 3.3 \\
\hline 77 & 28 & 3.3 & 1.3 \\
\hline 126 & 52 & 4.4 & 2.1 \\
\hline 234 & 108 & 7.5 & 4.1 \\
\hline 131 & 60 & 4.9 & 2.6 \\
\hline 175 & 66 & 6.2 & 2.2 \\
\hline 126 & 74 & 5.1 & 3.1 \\
\hline 247 & 98 & 6.2 & 3.1 \\
\hline 300 & 156 & 2.7 & 1.7 \\
\hline 156 & 76 & 1.7 & 1.1 \\
\hline 121 & 58 & 1.3 & 0.7 \\
\hline 169 & 103 & 2.2 & 1.2 \\
\hline 169 & 64 & 2.2 & 1 \\
\hline 64 & 55 & 1 & 0.8 \\
\hline 107 & 50 & 1.3 & 0.7 \\
\hline 80 & 50 & 1 & 0.7 \\
\hline 118 & 89 & 1.3 & 1 \\
\hline 118 & 95 & 1.9 & 1.3 \\
\hline 195 & 96 & 1.9 & 1 \\
\hline 298 & 123 & 3.8 & 2.4 \\
\hline 166 & 56 & 5.8 & 2.5 \\
\hline 136 & 61 & 5.8 & 3 \\
\hline 252 & 137 & 4.8 & 3 \\
\hline 177 & 107 & 3.6 & 2.5 \\
\hline 218 & 191 & 4.7 & 4.4 \\
\hline 191 & 82 & 4.4 & 2.1 \\
\hline 261 & 210 & 6.4 & 5.2 \\
\hline 210 & 90 & 5.2 & 2.6 \\
\hline 120 & 34 & 4.5 & 1.5 \\
\hline
\end{tabular}

Figura 1. Urea y creatinina en $\mathrm{mg} / \mathrm{dl}$ pre y post procedimiento con la técnica Sledd.

En nuestra experiencia en las técnicas continuas, cada 24 horas se gasta como mínimo un kit para monitor (aunque en teoría puede substituirse cada 48/72 horas), que cuesta unos 150 euros. Los líquidos de dializante y/o reposición, que son usados en estas técnicas cuestan de 7 a 15 euros cada unidad, debiendo ser sustituidos en un día de 4 a 5 veces; contamos por ultimo con los sacos de efluente con un costo de 6 euros cada uno, estas bolsas son también sustituidas cada 4 o 5 horas, lo que da un importe total aproximado de 235 euros por cada 24 horas.

\begin{tabular}{|l|c|c|c|c|c|}
\hline urea pre & urea $\mathbf{2 4} \mathbf{h}$ & tasa $\mathbf{2 4} \mathbf{h}$ & creatinina pre & creatinina $\mathbf{2 4} \mathbf{h}$ & tasa $\mathbf{2 4} \mathbf{h}$ \\
\hline 231 & 145 & $37 \%$ & 4.2 & 2.8 & $31 \%$ \\
\hline 179 & 98 & $45 \%$ & 3.9 & 2.3 & $39 \%$ \\
\hline 242 & 171 & $29 \%$ & 5.5 & 4.2 & $23 \%$ \\
\hline 154 & 104 & $32 \%$ & 6.1 & 4.3 & $29 \%$ \\
\hline 298 & 181 & $39 \%$ & 6.0 & 3.8 & $37 \%$ \\
\hline 255 & 170 & $33 \%$ & 5.7 & 3.9 & $31 \%$ \\
\hline
\end{tabular}

Tabla 2. Valores de urea y creatinina en $\mathrm{mg} / \mathrm{dl}$ con la técnica continua.

\begin{tabular}{|c||c|}
\hline \multicolumn{1}{|c|}{ SLEDD } & TÉCNICA CONTINUA \\
\hline Tasa media de aclaramiento & $\begin{array}{c}\text { Tasa media de aclaramiento } \\
\text { de urea: }\end{array}$ \\
$48,3 \%$ & $37 \%$ \\
\hline Tasa media de aclaramiento & Tasa media de aclaramiento \\
de creatinina: & de creatinina: \\
$43 \%$ & $31,7 \%$ \\
\hline
\end{tabular}

Tabla 3. Tasas medias de aclaración de urea y creatinina de ambas técnicas

\section{DISCUSIÓN Y CONCLUSIONES}

Las técnicas mixtas (SLEDD) demostraron que pueden ser eficaces en pacientes inestables e incluso en pacientes sometidos a soporte de aminas, además de demostrar que en términos de aclaramiento de urea y creatinina resultaron hasta más eficaces que las técnicas continuas.

En el plano económico la técnica de SLEDD resultó ser más ventajosa, ya que por cada procedimiento se gasta aproximadamente una décima parte de lo que se gasta en las técnicas continuas en 24 horas. Se ha de tener en cuenta que en este estudio económico no se ha incluido el gasto de la anticoagulación, ya que la administración de heparina fue mucho menor en la SLEDD (media de 4400 UI por técnica) que en la técnica continua (media de $12.000 \mathrm{UI}$ en 24 horas).

Por otra parte, según las opiniones de los enfermeros que participaron en la experiencia, la aceptación de la técnica SLEDD fue buena y había mayor disponibilidad a la hora de ejercer otros cuidados de enfermería a los pacientes, así como una percepción propia de menor sobrecarga laboral.

El uso de SLEDD evidenció ventajas importantes sobre las técnicas continuas de depuración en cuidados intensivos y por tanto, apostamos en que será cada vez más implementado en otras Unidades de Cuidados Intensivos en el futuro. 


\section{AGRADECIMIENTOS}

Agradecemos a todos los compañeros enfermeros de la unidad de cuidados intensivos del hospital Curry Cabral de Lisboa la buena disposición en la participación en este estudio que no podría haberse hecho realidad sin ellos. Agradecemos la gran ayuda prestada por el Doctor Paulo Marcelino, la Doctora Susan Marum y la Doctora Dora Sargento, todos ellos médicos de la U.C.I. del mismo hospital, por nunca negar un apoyo que resultó imprescindible para la realización de este trabajo, a todos ellos "MUITO OBRIGADO".

\section{BIBLIOGRAFIA}

1. Kumar VA, Depner TA. Daily Dialysis in the intensive care unit. Int J artificial organs 2002; 25:1031.
2. Schaepler C, Amerlin R, Manns M. High clearance continuous renal replacentment therapy with a modified dialysis machine. A J Kidney Dis 1999; 72:520.

3. Kumar VA, Craig M, Depner TA, Yeun NW. Extended daily dialysis: a new approach to renal replacentment for acute renal failure in the intensive care unit. A J Kidney Dis 2000; 36:294.

4. Sigler MH. Transports characteristics of the slow therapies: applications for achieving adequacy of dialysis in acute renal failure. Adv Renal repleace 1997; 4:68.

5. Lonneman G, Floege J, Kliem V. Extended daily veno-venous high flux haemodialysis in the patients with acute renal failure and multiple organ dysfunction syndrome using a single part bath dialysis system. Nephrol Dial Transplant 2000; 15:1189. 